\title{
Segunda observación de Daphnis nerii (Linnaeus, 1758) en el País Vasco después de 161 años (Lepidoptera: Sphingidae).
} Second record of Daphnis nerii (Linnaeus, 1758) in the Basque Country after 161 years (Lepidoptera: Sphingidae).

David Alday ${ }^{1}$, José Antonio Gainzarain²

\section{8}

\section{Resumen}

Un macho de Daphnis nerii (Linnaeus, 1758) fue observado en Vitoria-Gasteiz el 29.10.2016. Se trata de la segunda cita de la especie para el País Vasco, 161 años después de la primera, que tuvo lugar en Getxo en 1855.

Palabras clave: Daphnis nerii, Sphingidae, Lepidoptera, 2016, País Vasco, España.

\section{Abstract}

A male of Daphnis nerii (Linnaeus, 1758) was observed in Vitoria-Gasteiz on 29.10.2016. This is the second record of the species for the Basque Country, 161 years after the first one, which took place in Getxo in 1855.

Key words: Daphnis nerii, Sphingidae, Lepidoptera, 2016, Basque Country, Spain.

\section{Laburpena}

Daphnis nerii (Linnaeus, 1758) ar bat ikusi zen Vitoria-Gasteizen 2016.10.29an. Euskadin gertatzen den bigarren behaketa da, 1855ean Getxon lehenengoa egin zenetik 161 urte geroago.

Gako hitzak: Daphnis nerii, Sphingidae, Lepidoptera, 2016, Euskadi, Espainia.

1 Geotech Gestión Espacial S. L. Parque Tecnológico de Álava.

C/ Albert Einstein 44, edificio 6, oficina 901510 Miñano

2 Instituto Alavés de la Naturaleza.

Apdo. de correos 209201080 Vitoria-Gasteiz.

*Correspondencia: j.gainzarain@gmail.com 
Daphnis nerii (Linnaeus, 1758) es un lepidóptero de llamativo aspecto y gran tamaño, cuya distribución eminentemente paleotropical alcanza por el norte el entorno mediterráneo. En el sur de Europa la diferencia entre poblaciones residentes y migrantes está poco definida: existen poblaciones permanentes por ejemplo en Sicilia, Creta y Chipre, pero en el continente no mantiene una presencia estable ya que, aunque la especie llega a reproducirse, no soporta los inviernos rigurosos (Pittaway, 2020). Si bien aparece con cierta frecuencia como migradora en gran parte de Europa, en la península ibérica se conocen muy pocas observaciones de la especie, la mayoría de ellas en provincias del litoral mediterráneo (Monasterio León et al., 2018). En el País Vasco la única cita publicada hasta la fecha corresponde a varias orugas localizadas en agosto de 1885 en un jardín de Getxo (Bizkaia) (Seebold, 1898).

Así pues, la observación de un macho el 29.10.2016 en Vitoria-Gasteiz supone la segunda cita para el País Vasco 161 años después de la primera. El ejemplar en cuestión se localizó a las 19 h en el casco urbano de la ciudad (30T WN 2676043765525 m s.n.m.). Estaba posado en la entrada de un bar en una céntrica calle muy concurrida y pudo ser fotografiado con un teléfono móvil antes de alejarse volando (figura 1).

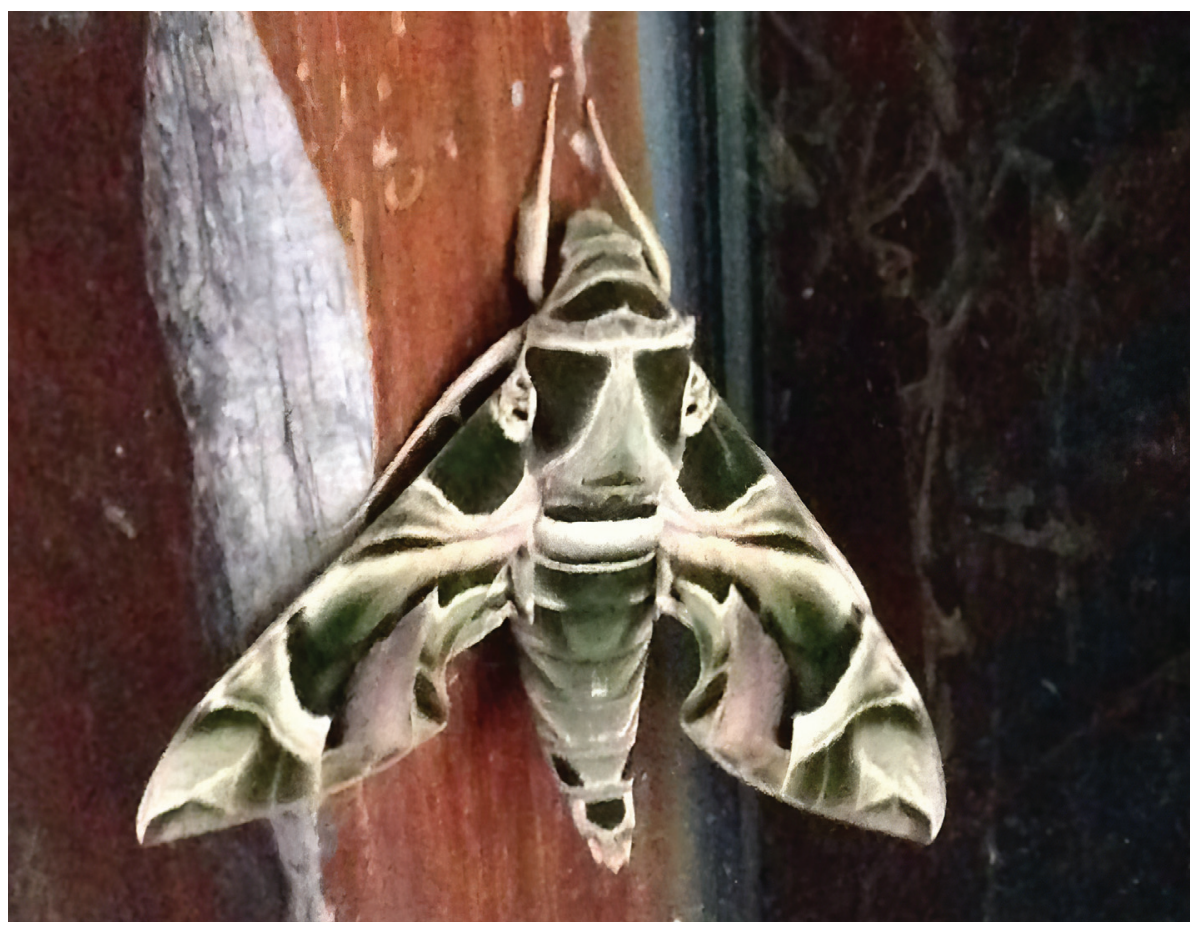

Fig. 1.- Macho de Daphnis nerii observado en Vitoria-Gasteiz el 29.10.2016 (fotografía de DA).

Fig. 1.- Daphnis nerii male observed in Vitoria-Gasteiz on 29.10.2016 (photograph by DA). 
No existen en las inmediaciones pies de su planta nutricia, la adelfa (Nerium oleander), una especie rara en los jardines de la ciudad (obs. pers.). No obstante, la aparente ausencia de desgaste en las alas sugiere que el ejemplar podría tener un origen local (Monasterio León et al., 2018).

Las observaciones de Daphnis nerii en España (no se ha registrado nunca en Portugal) siguen un peculiar patrón temporal: según las revisiones de Montagud-Alario y Engra (2017) y Monasterio León et al. (2018), se conocen unas pocas citas en el siglo XIX y las dos primeras décadas del XX, todas ellas en provincias costeras. Posteriormente, entre los años 50 y 70, fue localizada en tres ocasiones en la provincia de Girona (existe además una referencia en Jaén que se considera dudosa). Tras estos avistamientos se produjo un intervalo de cuarenta años sin que se registrara a la especie en la Península.

Esta ausencia de observaciones se prolongó hasta octubre de 2016, cuando, unos días antes de la cita que recoge la presente nota, se localizaron cinco larvas en un jardín urbano de Castellón (Montagud-Alario y Engra, 2017). La coincidencia temporal de las dos primeras citas para la especie en la península ibérica en el siglo XXI se relaciona con los numerosos avistamientos que tuvieron lugar en diferentes lugares de Europa durante el otoño de 2016, lo que sugiere la existencia de una irrupción de cierta magnitud. Así, la especie se detectó con frecuencia superior a la habitual por ejemplo en Alemania (Trusch, 2016), Francia (Haxaire, 2020), Países Bajos y Bélgica (Vermandel y Vliegenthart, 2017). Asimismo, en Malta se registraron las primeras citas de la especie desde 1998 (Catania, 2019).

Es llamativo que solo dos años después, se produjera en otoño de 2018 una nueva irrupción de Daphnis nerii en Europa occidental, al parecer de mayor entidad que la de 2016 (Catania, 2019; Haxaire, 2020). En España este movimiento se tradujo en una proliferación de avistamientos sin precedentes. Cuatro de ellos tuvieron lugar en Cataluña (Guzmán, 2018; Monasterio León et al., 2018) y se obtuvieron las primeras citas de la especie para Cantabria (Pardo de Santayana Sanz y Pardo de Santayana Trueba, 2019), islas Baleares (dos observaciones diferentes; Truyols-Henares et al., 2019), Extremadura, La Rioja, Murcia y Navarra (Monasterio León et al., 2018). La excepcionalidad de esta cantidad de registros (dos en 2016 y once en 2018) se pone de manifiesto si se compara con los seis obtenidos a lo largo de todo el siglo XX en España. Queda por comprobar si se trata de un fenómeno aislado o del inicio de un periodo de aparición regular de Daphnis nerii en la península ibérica.

\section{Agradecimientos}

A Fernando Jubete, por su ayuda en la búsqueda de bibliografía, y a Yeray Monasterio, que con sus comentarios contribuyó a mejorar una primera versión de la presente nota. 


\section{Bibliografía}

Catania, A., 2019. Note of new and past records of Daphnis nerii (Linnaeus, 1758) from Malta (Lepidoptera: Sphingidae). Shilap Rev. Lepidopterol. 47(185), 185-188.

Guzmán, E., 2018. Migració de Daphnis nerii (Linnaeus, 1758) (Sphingidae) a Catalunya. Butll. Soc. Catalana. Lepidopterol. 109, 101-103.

Haxaire, J., 2020. Les sphingidae de France. Available from: http://sphingidaehaxaire.com/index.php/macroglossinae-2/daphnis-nerii/. [accessed 28.08.2020].

Monasterio León, Y., Iglesias Baquero, A., Escobés Jiménez, R., 2018. Cinco nuevas observaciones de Daphnis nerii (Linnaeus, 1758) en España (Lepidoptera: Sphingidae). Bol. SEA 63, 295-296.

Montagud-Alario, S., Engra, M.A., 2017. Nuevo registro de Daphnis nerii en la Península Ibérica (Lepidoptera: Sphingidae). Bol. SEA 60, 369-371.

Pardo de Santayana Sanz, J.M., Pardo de Santayana Trueba, G., 2019. Nuevo registro de Daphnis nerii (Linnaeus, 1758) para la Península Ibérica (Lepidoptera: Sphingidae). Arquivos Entomolóxicos 21, 145-146.

Pittaway, A.R., 2020. Sphingidae of the Western Palaearctic (including Europe, North Africa, the Middle East, western Siberia and western Central Asia). Available from: http://tpittaway.tripod.com/sphinx/list.htm [accessed 24.08.2020].

Seebold, T., 1898. Catalogue raisonné des lépidoptères des environs de Bilbao (Espagne). An. Soc. Esp. Hist. Nat. 27, 111-175.

Trusch, R., 2016. Aktuelle Funde des Oleanderschwärmers Daphnis nerii (Linnaeus, 1758) in Baden-Württemberg (Lepidoptera: Sphingidae). Carolinea 74, 123-128.

Truyols-Henares, F., Canyelles, X., Febrer-Serra, M., Perelló E., Pinya, S., 2019. First records of the Oleander hawkmoth Daphnis nerii (Linnaeus, 1758) from the Balearic Islands, Spain (Lepidoptera: Sphingidae). Shilap Rev. Lepidopterol. 47(185), 25-28.

Vermandel, E., Vliegenthart, A., 2017. Trekvlinders in België en Nederlandin 2016 (Lepidoptera). Phegea 45, 98-104.

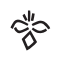

Fecha de recepción/ Date of reception: 01/09/2020

Fecha de aceptación / Date of acceptance: 01/10/2020

Editor Asociado / Associate editor: Alberto Castro 